\title{
0403 INJURY AND SPATIAL EPIDEMIOLOGY OF SEVERE ADULT TRAUMA: IMPLICATIONS FOR PREVENTION
}

T Charyk-Stewart*, D A Tanner, J Gilliland, M Healy, J Williamson, S McKenzie, M J Girotti, D D Fraser Correspondence: London Health Sciences Centre, University of Western Ontario, Trauma Program, Rm E1-129, 800 Commissioners Road East, PO Box 5010 London, Ontario, N6A 5W9, Canada

10.1136/ip.2010.029215.403

Background Identifying who is injured within a geographic region, by what mechanism, is essential for injury prevention (IP). Our objective was to define the injury and spatial epidemiology of severe adult traumas to prioritise and target initiatives.

Methods Epidemiologic profiles were generated for severely injured (ISS $>12)$ adult ( $\geq 18$ years) patients treated at Lead Trauma Hospitals (LTH) in Southwestern Ontario, 2004-2009. Sub-analysis was undertaken by age groups (18-24; 25-64; $65+$ years). Injury cases were mapped by patient residence and place of injury to examine spatial relationships.

Results LTHs resuscitated 2804 severely injured adults (15\% young adult, $55 \%$ adult, $30 \%$ senior; $72 \%$ male). Patient residences were dispersed throughout SWO, with clusters in cities and lower-income areas. MVCs accounted for $61 \%$ and $46 \%$ of injuries among young adults and adults, respectively. Only $60 \%$ of injured-occupants wore a seatbelt; $24 \%$ of drivers had a BAC above the legal limit. MVCs were overly concentrated on high-density urban areas with highly mixed land uses. Alcohol was involved with nearly one-third of nonsenior severe injury ( $48 \%$ of assaults; $34 \%$ of crashes). Falls were the leading injury mechanism for seniors (68\%); $67 \%$ occurred at home. Only $6 \%$ of patients were injured at work, half involved falls. Mortality was $15 \%$, with $42 \%$ fall-related deaths.

Conclusion Integrating injury epidemiology with geographic data on patients daily surroundings allowed for the identification of socio-spatial variations in injury patterns among vulnerable groups. This approach identified MVCs, falls and alcohol use as IP priorities to be targeted to the populations and regions of greatest need. 